\title{
mRNA degradation by miRNAs and GW182 requires both CCR4:NOT deadenylase and DCP1:DCP2 decapping complexes
}

\author{
Isabelle Behm-Ansmant, ${ }^{1,4}$ Jan Rehwinkel, ${ }^{1,4}$ Tobias Doerks, ${ }^{1}$ Alexander Stark, ${ }^{3}$ Peer Bork, ${ }^{1}$ \\ and Elisa Izaurralde ${ }^{1,2,5}$ \\ ${ }^{1}$ European Molecular Biology Laboratory (EMBL), D-69117 Heidelberg, Germany; ${ }^{2}$ Max-Planck-Institute for Developmental \\ Biology, D-72076 Tübingen, Germany
}

\begin{abstract}
MicroRNAs (miRNAs) silence the expression of target genes post-transcriptionally. Their function is mediated by the Argonaute proteins (AGOs), which colocalize to P-bodies with mRNA degradation enzymes.

Mammalian P-bodies are also marked by the GW182 protein, which interacts with the AGOs and is required for miRNA function. We show that depletion of GW182 leads to changes in mRNA expression profiles strikingly similar to those observed in cells depleted of the essential Drosophila miRNA effector AGO1, indicating that GW182 functions in the miRNA pathway. When GW182 is bound to a reporter transcript, it silences its expression, bypassing the requirement for AGO1. Silencing by GW182 is effected by changes in protein expression and mRNA stability. Similarly, miRNAs silence gene expression by repressing protein expression and/or by promoting mRNA decay, and both mechanisms require GW182. mRNA degradation, but not translational repression, by GW182 or miRNAs is inhibited in cells depleted of CAF1, NOT1, or the decapping DCP1:DCP2 complex. We further show that the N-terminal GW repeats of GW182 interact with the PIWI domain of AGO1. Our findings indicate that GW182 links the miRNA pathway to mRNA degradation by interacting with AGO1 and promoting decay of at least a subset of miRNA targets.
\end{abstract}

[Keywords: Argonaute; deadenylation; decapping; GW182; mRNA decay; P-bodies]

Supplemental material is available at http://www.genesdev.org.

Received February 27, 2006; revised version accepted May 30, 2006.

RNA silencing pathways are conserved, and regulate gene expression at both transcriptional and post-transcriptional levels. These pathways are triggered by the presence of double-stranded RNAs (dsRNAs) of diverse origin, which are processed into small RNAs of 20-30 nucleotides in length. MicroRNAs (miRNAs) are a specific class of genome-encoded small RNAs that regulate gene expression post-transcriptionally (Bartel 2004; Filipowicz 2005). We now know of hundreds of miRNAs in different organisms, and each is thought to regulate the expression of hundreds of targets. They are implicated in biological processes as diverse as cell differentiation and proliferation, apoptosis, metabolism, and development (Bartel 2004; Filipowicz 2005).

${ }^{3}$ Present addresses: Broad Institute of Massachusetts Institute of Technology and Harvard, Cambridge, MA 02141, USA; Computer Science and Artificial Intelligence Laboratory, Massachusetts Institute of Technology, Cambridge, MA 02139, USA.

${ }^{4}$ These authors contributed equally to this work.

${ }^{5}$ Corresponding author.

E-MAIL elisa.izaurralde@tuebingen.mpg.de; FAX 49-7071-601-1353. Article published online ahead of print. Article and publication date are online at http://www.genesdev.org/cgi/doi/10.1101/gad.1424106.
To accomplish their regulatory function miRNAs associate with the Argonaute proteins to form RNA-induced silencing complexes (RISCs), which elicit decay or translational repression of complementary mRNA targets (Bartel 2004; Filipowicz 2005). In plants, miRNAs are often fully complementary to their targets, and elicit mRNA decay. In contrast, animal miRNAs are only partially complementary to their targets, and silence gene expression by mechanisms that remain elusive (Filipowicz 2005). Recent studies have shown that miRNAs silence gene expression by inhibiting translation initiation at an early stage involving the cap structure, as mRNAs translated via cap-independent mechanisms escape miRNA-mediated silencing (Humphreys et al. 2005; Pillai et al. 2005). Other studies have suggested that translation inhibition occurs after initiation, based on the observation that miRNAs and some targets remain associated with polysomes (Olsen and Ambros 1999; Kim et al. 2004; Nelson et al. 2004; Petersen et al. 2006). In addition, animal miRNAs can also induce significant degradation of mRNA targets despite imperfect mRNAmiRNA base-pairing (Bagga et al. 2005; Jing et al. 2005; 
Lim et al. 2005; Giraldez et al. 2006; Rehwinkel et al. 2006; Wu et al. 2006).

The existence of a link between the miRNA pathway and mRNA decay is supported by the observation that mammalian Argonaute proteins (AGO1-AGO4), miRNAs, and miRNA targets colocalize to cytoplasmic foci known as P-bodies (Jakymiw et al. 2005; Liu et al. 2005a,b; Meister et al. 2005; Pillai et al. 2005; Sen and Blau 2005). These mRNA processing bodies are discrete cytoplasmic domains where proteins required for bulk mRNA degradation in the 5'-to-3' direction accumulate (e.g., the decapping DCP1:DCP2 complex and the 5' -to3' exonuclease XRN1) (for review, see Valencia-Sanchez et al. 2006). Additional components of P-bodies in yeast and/or human cells include the CCR4:NOT deadenylase complex, auxiliary decapping factors (e.g., the LSm1-7 complex and Patlp/Mtrlp), the cap-binding protein eIF4E, and the RNA helicase Dhh1/Me31B involved in translational repression (Valencia-Sanchez et al. 2006). In metazoa, P-bodies are also marked by the presence of GW182, a protein with glycine-tryptophan repeats /GW repeats) required for P-body integrity (Eystathioy et al. 2002, 2003).

The presence of Argonaute proteins, miRNAs, and miRNA targets in P-bodies has led to a model in which translationally silenced mRNAs are sequestered to these bodies, where they may undergo decay (Jakymiw et al. 2005; Liu et al. 2005a,b; Meister et al. 2005; Pillai et al. 2005; Rehwinkel et al. 2005; Sen and Blau 2005). At present, it is unclear whether the localization in P-bodies is the cause or consequence of the translational repression, though several lines of evidence point to a direct role for P-body components in miRNA-mediated gene silencing. First, DCP1, GW182, and its paralog TNRC6B associate with AGO1 and AGO2 in human cells (Jakymiw et al. 2005; Liu et al. 2005a,b; Meister et al. 2005; Sen and Blau 2005). Second, depletion of GW182 in human cells impairs both miRNA function and mRNA decay triggered by complementary short interfering RNAs (siRNAs) (Jakymiw et al. 2005; Liu et al. 2005b; Meister et al. 2005). Similarly, miRNA function is impaired in Drosophila Schneider cells (S2 cells) depleted of GW182 or the decapping DCP1:DCP2 complex (Rehwinkel et al. 2005). Finally, the Caenorhabditis elegans protein AIN-1, which is related to GW182, is required for gene regulation by at least a subset of miRNAs (Ding et al. 2005).

In Drosophila, siRNA-guided endonucleolytic cleavage of mRNAs (RNA interference [RNAi]) is mediated by AGO2, while gene silencing by miRNAs is mediated by AGO1 (Okamura et al. 2004; Rand et al. 2004; Miyoshi et al. 2005; Rehwinkel et al. 2005). That siRNAs and miRNAs enter separate pathways in Drosophila is further supported by the observation that depletion of GW182 inhibits miRNA-mediated, but not siRNA-mediated gene silencing (Rehwinkel et al. 2005). The precise role of GW182 in the miRNA pathway is unknown. GW182 could have an indirect role by affecting P-body integrity. Alternatively, it could be more directly involved, localizing miRNA targets to P-bodies or facilitating the mRNP remodeling steps required for the si- lencing and/or decay of these targets (Jakymiw et al. 2005; Liu et al. 2005b; Meister et al. 2005; Rehwinkel et al. 2005).

In this study, we further investigate the role of Drosophila GW182 in the miRNA pathway. We show that depletion of GW182 leads to changes in mRNA expression profiles strikingly similar to those observed in cells depleted of AGO1, indicating that GW182 is a genuine component of the miRNA pathway. In cells in which miRNA-mediated gene silencing is suppressed by depletion of AGO1, GW182 can still silence the expression of bound mRNAs, suggesting that GW182 acts downstream of AGO1. We further show that GW182 triggers silencing of bound transcripts by inhibiting protein expression and promoting mRNA decay via a deadenylation and decapping mechanism. Finally, we provide evidence that mRNA degradation by miRNAs requires GW182, the CCR4:NOT deadenylase, and the DCP1:DCP2 decapping complexes. Together with the observation that GW182 interacts with AGO1, our results indicate that binding of GW182 to miRNA targets induces silencing and can trigger mRNA degradation, providing an explanation for the observed changes in mRNA levels, at least for a subset of animal miRNA targets.

\section{Results}

\section{Drosophila GW182 functions in the miRNA pathway}

To investigate whether GW182 functions in the miRNA pathway or has additional roles in mRNA turnover, we compared RNA expression profiles of GW182-depleted cells with those of AGO1- or AGO2-depleted cells, using high-density oligonucleotide microarrays. For each protein, we obtained expression profiles from two or three independent RNA samples. As a reference, RNA samples were isolated from mock-treated cells. We also examined mRNA profiles in cells treated with GFP (green fluorescent protein) dsRNA to identify mRNAs that are likely to be regulated nonspecifically in response to the dsRNA treatment.

Western blot analysis indicated that the residual levels of the proteins in depleted cells were $<10 \%$ of those observed in control cells (Fig. 1A, lanes 5,12 vs. 1,7). Importantly, depletion of AGO1 does not affect the expression of GW182 or AGO2 (Fig. 1A, lane 11; data not shown). Conversely, GW182 depletion had no effect on AGO1 or AGO2 expression levels (Fig. 1A, lane 6; data not shown).

The profiles displayed by cells depleted of GW182 or AGO1 were strikingly similar (Fig. 1B). The rank correlation coefficient $r$ between GW182 and AGO1 profiles was 0.92 , indicating that these proteins regulate common targets. A subset of these targets was also regulated by AGO2 (Fig. 1B). The rank correlation coefficient was 0.64 between GW182 and AGO2 profiles, and 0.63 between AGO1 and AGO2. Indeed, we have shown before that AGO2 affects the expression of a subset of transcripts regulated by AGO1, and that only a few transcripts are exclusively regulated by $\mathrm{AGO} 2$ in $\mathrm{S} 2$ cells 
(Rehwinkel et al. 2006). For this reason AGO2 profiles were not considered further.

To investigate the similarity of the cellular response to the depletion of GW182 and AGO1, we selected mRNAs belonging to specific classes in the GW182 knockdown (at least twofold overrepresented or twofold underrepresented, respectively) and analyzed their levels in the

A

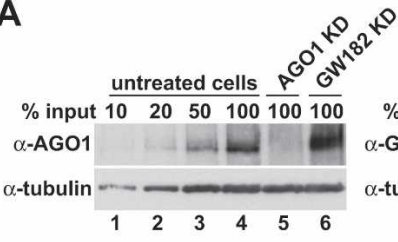

B
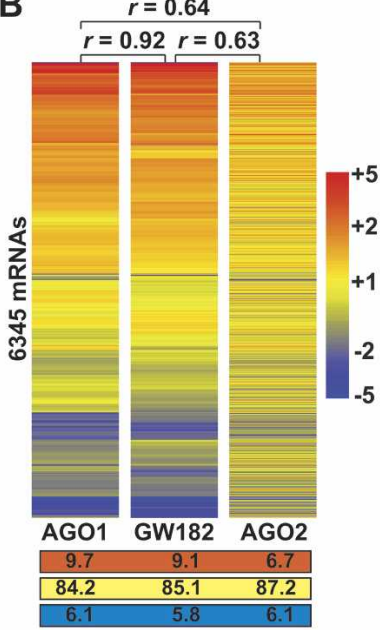

$\mathrm{C}_{2 \text {-fold up or down }}$ in GW182

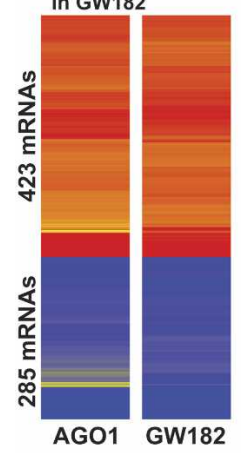

G Exclusively regulated

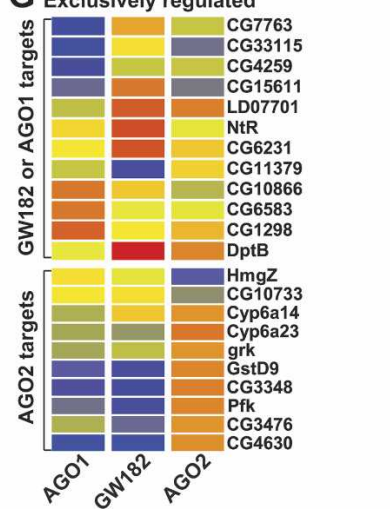

AGO1 knockdown (Fig. 1C). There was a high degree of overlap between regulated transcripts in the two knockdowns (Fig. 1C; Supplementary Table 1). Indeed, among the 423 mRNAs that were at least twofold overrepresented in the GW182 knockdown, 75.8\% changed levels concordantly in AGO1-depleted cells and were at least twofold up-regulated (Fig. 1C). Conversely, among the 486 mRNAs that were at least twofold overrepresented in the AGO1 knockdown, 66\% were twofold up-regulated in the GW182 knockdown (Fig. 1D; Supplementary Table 1). Down-regulated transcripts overlapped to a similar extent (Fig. 1C,D; Supplementary Table 1).

Transcripts up-regulated in AGO1-depleted cells are significantly enriched in predicted miRNA targets (Rehwinkel et al. 2006). We tested the enrichment for targets of cloned, nonredundant miRNAs (i.e., unique seed sequences) predicted by Stark et al. (2005). We observed a significant enrichment for predicted miRNA targets among transcripts up-regulated in the GW182 knockdown $\left(P=6 \times 10^{-20}\right)$ and AGO1 knockdown $\left(P=3.2 \times 10^{-32}\right)$. Consistently, the levels of detectable, experimentally validated miRNA targets were at least twofold up-regulated in cells depleted of GW182 or AGO1 (Fig. 1E). We validated the array data for a subset of predicted miRNA targets by Northern blot (Fig. 1F; data not shown). Downregulated transcripts were not significantly enriched for predicted targets $(P \sim 1)$, suggesting that these transcripts are unlikely to be directly regulated by the miRNA pathway.

To determine whether GW182 has additional func-

Figure 1. Expression profiles of Drosophila S2 cells depleted of GW182, AGO1, or AGO2. (A) S2 cells were treated with the dsRNAs indicated above the lanes. The effectiveness of the depletions was analyzed by Western blot with the antibodies indicated on the left. In lanes 1-4, dilutions of samples isolated from control cells were loaded to assess the efficacy of the depletion. Antibodies against tubulin were used as a loading control. (KD) Knockdowns. (B) Comparison of the average expression levels of the 6345 detectable transcripts in the profiles obtained for AGO1, GW182, or AGO2. The rank correlation coefficient of the profiles $(r)$ is indicated. The table shows the percentage of transcripts at least twofold overrepresented (red), between twofold up- and down-regulated (yellow), or at least twofold underrepresented (blue). (C) Expression profiles of RNAs at least twofold overrepresented or underrepresented in the two independent profiles obtained for GW182 (Supplementary Table 1). (D) Expression profiles of RNAs at least twofold overrepresented or underrepresented in the two profiles obtained for AGO1 (Supplementary Table 1). (E) Expression profiles of validated miRNA targets. Asterisks indicate endogenous targets used to generate the miRNA reporters shown in Figure 5. $(F)$ Validation of microarray results by Northern blot analysis. The identity of the selected transcripts and the predicted cognate miRNAs are indicated on the right. The signals from the Northern blot were normalized to rp49 mRNA (not shown). These values are compared with the values measured by microarray. Values are given as fold changes relative to the values obtained in mock-treated (cont.) cells. (G) Transcripts at least twofold regulated in either AGO1 or GW182 samples and $<1.3$-fold changed or inversely correlated in GW182 or AGO1 samples, respectively, and transcripts regulated exclusively by AGO2. 
tions in mRNA turnover, we sought transcripts regulated exclusively in GW182-depleted cells but clearly unaffected (changed $<1.3$-fold) or even showing inverse correlation in the AGO1 knockdown. Only six transcripts were found to be regulated exclusively in GW182depleted cells (Fig. 1G).

We also analyzed whether transcripts exclusively regulated by AGO2 but not by the miRNA pathway (as described by Rehwinkel et al. 2006) were affected by GW182 depletion. We found that these transcripts were not regulated by GW182 (Fig. 1G), a result consistent with the possibility that AGO2 regulates the expression of these transcripts by a mechanism involving endogenous siRNAs, whose function is not affected in GW182depleted cells (Rehwinkel et al. 2005). To summarize, GW182 and AGO1 regulate a common set of mRNAs that are significantly enriched in predicted and validated miRNA targets, indicating that these proteins act in the miRNA pathway; a subset of these targets is also regulated by AGO2, as reported before (Rehwinkel et al. 2006).

\section{GW182 belongs to a conserved family of proteins} present only in metazoa

To identify potential functional domains in GW182 we compared orthologous sequences across species. PSIBLAST searches (Altschul et al. 1997) using Drosophila and human GW182 as input sequences (Eystathioy et al. 2002; Rehwinkel et al. 2005) led to the identification of single genes encoding GW182 orthologs in Drosophila melanogaster, Anopheles gambiae, and Apis mellifera genomic sequences, and three paralogous genes in Danio rerio, Gallus gallus, Mus musculus, Rattus norvegicus, and Homo sapiens, suggesting that duplication of the GW182 gene occurred after the divergence of invertebrates and vertebrates. The three paralogs in vertebrates are known as trinucleotide repeat containing (TNRC) proteins 6A, 6B, and 6C. TNRC6A corresponds to GW182 (Eystathioy et al. 2002; Meister et al. 2005).

The GW182 protein family is characterized by the presence of a central ubiquitin-associated (UBA) domain and a C-terminal RNA recognition motif (RRM) (Fig. 2A). Furthermore, three distinctive blocks of glycinetryptophan repeats (referred to as $\mathrm{N}$-terminal, middle, and C-terminal GW repeats) can be found, as well as a glutamine-rich (Q-rich) region located between the UBA domain and the RRM (Fig. 2A). The RRM and the Nterminal GW repeats have been described before (Eystathioy et al. 2002). The UBA domain was not found above the significance threshold in all family members, but iterative PSI-BLAST searches unambiguously confirmed the UBA homology for all family members.

In addition, a multiple sequence alignment of the protein family sequences revealed two highly conserved motifs (I and II) of $\sim 50$ residues within the $\mathrm{N}$-terminal GW repeats (Fig. 2A). The insect proteins are shorter than the vertebrate orthologs, and start exactly with motif I (Fig. 2A).
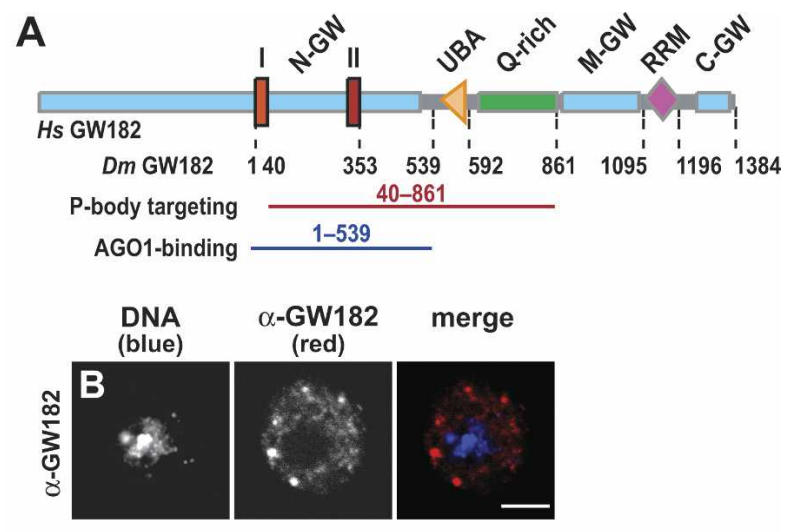

DNA
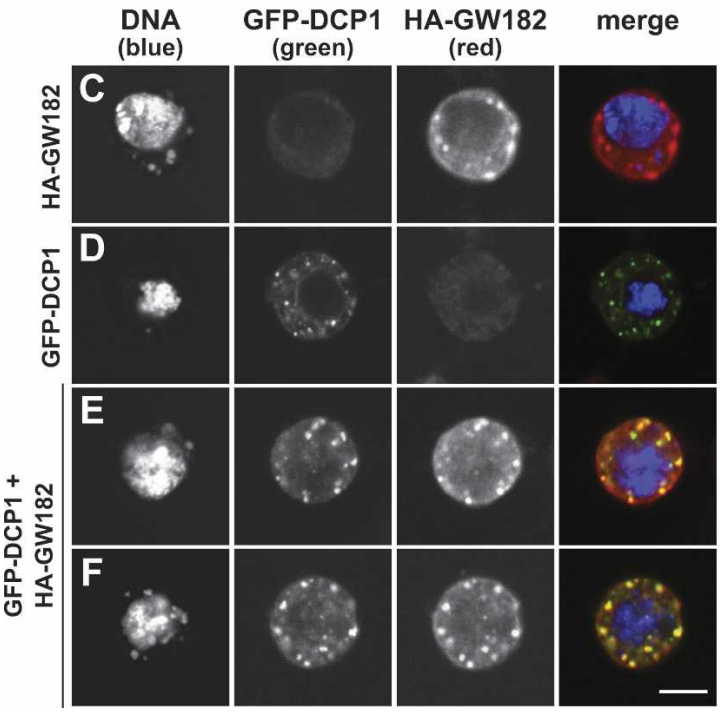

Figure 2. The N-terminal domain of GW182 is required for P-body targeting. (A) Domain architecture of GW182 and related proteins. (N-GW) N-terminal GW repeats; (M-GW) middle GW repeats; (C-GW) C-terminal GW repeats; (UBA) ubiquitinassociated domain; (Q-rich) region rich in glutamine; (RRM) RNA recognition motif. Orange and red boxes I and II indicate two conserved motifs within the $\mathrm{N}$-terminal GW repeats. Numbers under the protein outline represent amino acid positions at fragment boundaries for the Drosophila protein. The protein domains sufficient for the localization to P-bodies and the interaction with AGO1 are indicated. (B) Confocal fluorescent micrograph of S2 cells stained with anti-GW182 antibodies. (CF) Confocal fluorescent micrographs of $\mathrm{S} 2$ cells expressing HAGW182 and/or GFP-DCP1. Bar, $5 \mu \mathrm{m}$.

\section{Motif II, the UBA, and the Q-rich domains are required for the localization of GW182 in cytoplasmic foci}

We examined the subcellular localization of Drosophila GW182 in S2 cells using an antibody raised against the RRM domain. The GW182 signal was detected throughout the cytoplasm and in discrete cytoplasmic foci of varying number and size (Fig. 2B). Similarly, transiently expressed hemagglutinin (HA)- or GFP-tagged GW182 localized in cytoplasmic foci, indicating that the localization of these proteins reflects that of endogenous GW182 (Fig. 2C; data not shown). To investigate whether these 
GW182 foci are related to P-bodies, we transfected S2 cells with HA-GW182 and GFP fusions of known P-body components (e.g., DCP1, DCP2, and Me31B). HAGW182 colocalized with the GFP-tagged proteins in cytoplasmic foci (Fig. 2E,F; data not shown), suggesting that these foci represent P-body-like structures. Analysis of the subcellular localization of HA-GW182 protein fragments indicate that the $\mathrm{N}$-terminal GW repeats including motif II, the UBA, and the Q-rich domains all contribute to the localization of GW182 in cytoplasmic foci, though none of these domains suffice on their own (Fig. 2A; Supplementary Fig. S1).

The N-terminal GW repeats of GW182 interact with the PIWI domain of AGO1

In human cells, GW182 copurifies with Argonaute proteins and promotes their recruitment to P-bodies (Jaky- miw et al. 2005; Liu et al. 2005a,b; Meister et al. 2005; Sen and Blau 2005). The protein domains mediating the interaction between GW182 and AGOs have not been defined in previous studies. We observed that Drosophila AGO1 coimmunoprecipitated with HA-GW182 from S2 cell lysates, but not with the negative control, an HA fusion of maltose-binding protein (MBP) (Fig. 3A, lanes 9,10). Moreover, GFP-AGO1 spreads throughout the cytoplasm in transiently transfected S2 cells, but accumulates in cytoplasmic foci in cells coexpressing HAGW182 (Fig. 3, B vs. C,D). GFP-AGO1 was recruited efficiently to the foci formed by the N-terminal domain of GW182 (amino acids 1-861) (Fig. 3E), and endogenous AGO1 coimmunoprecipitated with this fragment (Fig. 3A, lane 11). C-terminal GW182 fragments (e.g., amino acids 861-1384) do not localize in cytoplasmic foci (Supplementary Fig. S1), and neither interact with AGO1 nor induce its accumulation in foci when coexpressed
A
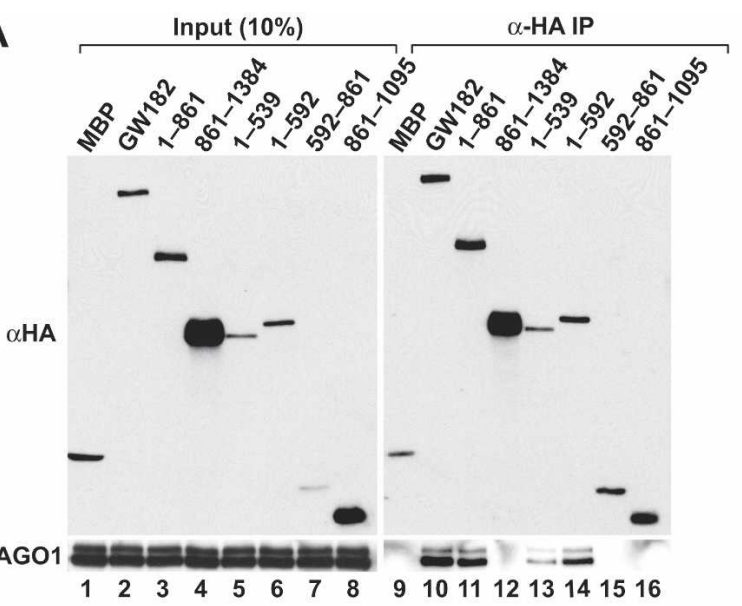

H

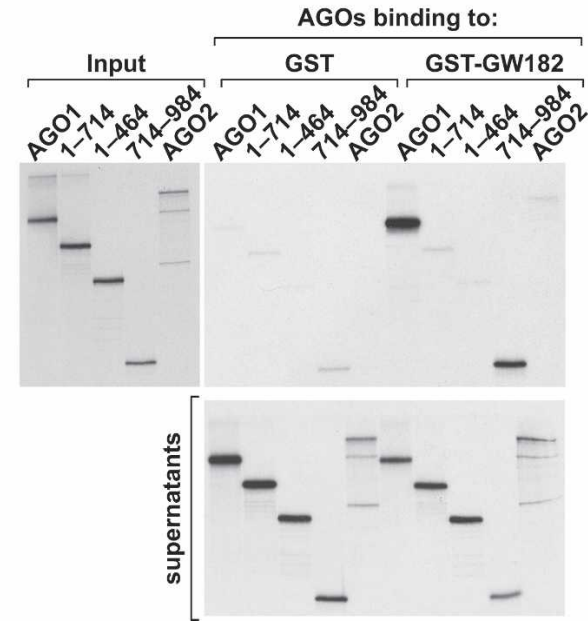

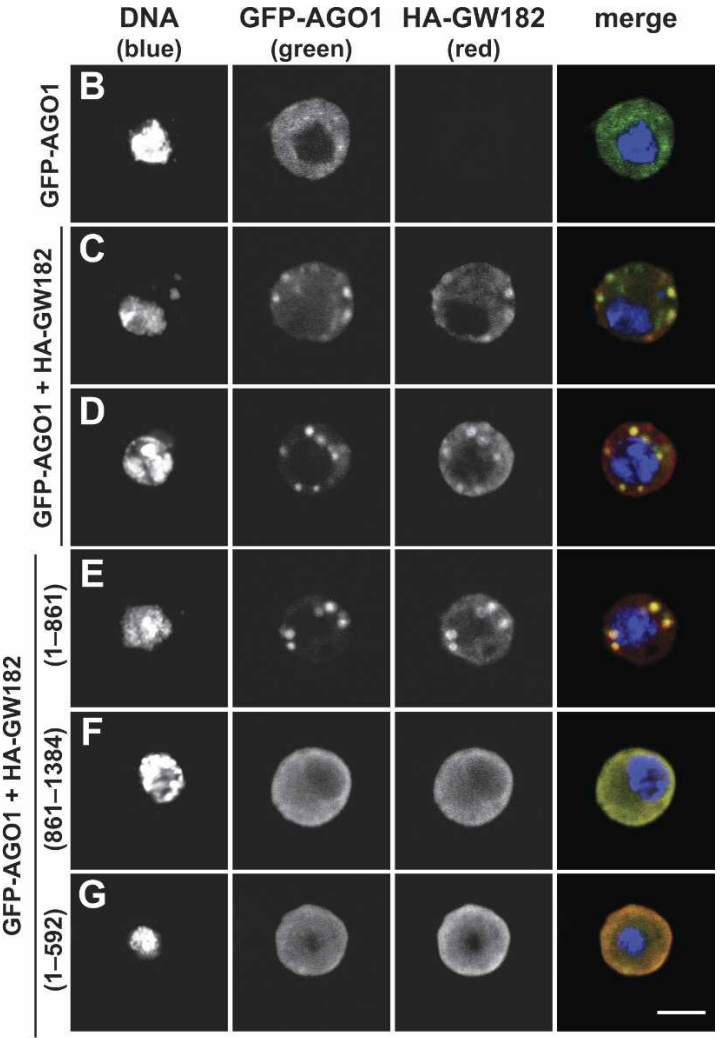

Figure 3. The N-terminal domain of GW182 interacts with the PIWI domain of AGO1. (A) HA-GW182 or the indicated protein fragments were transiently expressed in S2 cells. Cell lysates were immunoprecipitated using anti-HA antibodies. HA-MBP served as a negative control. Inputs and immunoprecipitates were analyzed by Western blot using anti-HA or anti-AGO1 antibodies. (B-G) Epitope-tagged GFP-AGO1 was expressed in S2 cells. In $C-G$, the effect of cotransfecting HA-tagged versions of GW182 or the indicated protein fragments on the localization of AGO1 was examined. The merged images show the GFP signal in green, the HA signal in red, and DNA in blue. Bar, $5 \mu \mathrm{m}$. (H) GST pull-down assays were performed with $\left[{ }^{35} \mathrm{~S}\right]$ methionine-labeled full-length AGO1, AGO2, or the indicated AGO1 protein fragments, and recombinant GST or GST-GW182 (fragment 1-592). Samples were analyzed by SDS-PAGE followed by fluorography. 
(Fig. 3A [lanes 12,16], F). We conclude that it is the Nterminal domain of GW182 that associates with AGO1 in vivo.

Further analysis indicates that fragments 1-592 and 1-539 are sufficient to coimmunoprecipitate AGO1, while the Q-rich domain, which is required for the localization to P-body-like structures, is inessential (Fig. 3A, lanes 13-15). Thus, binding to AGO1 and localization to cytoplasmic foci are mediated by partially overlapping but distinct domains (Fig. 2A). The recruitment of GFP-AGO1 to GW182 foci is not due to an unspecific aggregation of overexpressed interacting partners because GW182 fragments 1-592 and 1-539, which interact with AGO1 but do not form foci, do not induce the aggregation of GFP-AGO1 in the cytoplasm (Fig. 3G; data not shown). Moreover, GFP-AGO1 is not recruited to cytoplasmic foci formed by various P-body components including DCP2 or Ge-1 (Supplementary Fig. S1; data not shown).

To define the domain of AGO1 interacting with GW182, we performed pull-down experiments using recombinant GW182 fragment 1-592 fused to glutathione S-transferase and in vitro translated AGO1 (either full-length or lacking the PIWI domain [amino acids 1-714]). We also tested AGO1 fragments lacking both the PIWI and middle domains (amino acids 1-464), or comprising the PIWI domain alone (amino acids 714984). We observed that the PIWI domain of AGO1 was necessary and sufficient for its interaction with GW182 (Fig. 3H).

Remarkably, GW182 did not interact detectably with AGO2 in pull-down or coimmunoprecipitation assays (Fig. $3 \mathrm{H}$; data not shown). The specific interaction of GW182 with AGO1, but not AGO2, is consistent with a

Figure 4. GW182 silences the expression of bound transcripts. (A) Schematic representation of the F-Luc-5BoxB tethering reporter and of the F-Luc reporter control. $(B, C) \mathrm{S} 2$ cells were transfected with the F-Luc-5BoxB reporter or the F-Luc control, a plasmid expressing Renilla luciferase, and vectors expressing the $\lambda \mathrm{N}$-peptide or $\lambda \mathrm{N}$-GW182. Firefly luciferase activity was normalized to that of Renilla and set to 100 in cells expressing the $\lambda \mathrm{N}$-peptide alone. Mean values \pm standard deviations from four independent experiments $(n=4)$ are shown. In $C$, the corresponding RNA samples were analyzed by Northern blot. $(D)$ F-Luc-5BoxB or F-Luc mRNA levels were quantitated and normalized to the R-Luc transfection control in four independent experiments, including that shown in $C$. Normalized F-Luc mRNA levels in cells expressing the $\lambda \mathrm{N}$ peptide alone were set to $100 \%$. Mean values are shown. Error bars represent standard deviations. $(E)$ The normalized values of firefly luciferase activity shown in $B$ were divided by the normalized mRNA levels shown in $D$ to estimate the net effect of tethering GW182 on protein synthesis. $(F, G)$ S2 cells were transfected with plasmids for the $\lambda \mathrm{N}$-tethering assay. Cells were harvested at the indicated time points after addition of actinomycin $\mathrm{D}$. The decay of the F-Luc-5BoxB mRNA was monitored in cells expressing the $\lambda \mathrm{N}$ peptide $(F)$ or $\lambda$ N-GW182 $(G)$. (H) The levels of the F-Luc-5BoxB mRNA normalized to rp49 mRNA in three independent experiments are plotted against time. mRNA half-lives $\left(t_{1 / 2}\right)$ calculated from the decay curves are indicated. role for GW182 in the miRNA, but not the siRNA pathway (Rehwinkel et al. 2005).

\section{GW182 silences the expression of bound mRNAs}

To shed light on the role of GW182 in the miRNA pathway, we investigated whether binding of this protein to an mRNA is sufficient to silence its expression. To this end, we made use of the tethering assay described by Pillai et al. (2004). This assay involves the expression of $\lambda \mathrm{N}$-fusion proteins that bind with high affinity to five BoxB sites (5BoxB) in the 3 ' untranslated region (UTR) of a luciferase reporter mRNA (F-Luc-5BoxB reporter) (Fig. 4A).

S2 cells were transiently transfected with the F-Luc5 BoxB reporter, a plasmid expressing GW182 fused to the $\lambda \mathrm{N}$-peptide ( $\lambda \mathrm{N}-\mathrm{GW} 182)$, and a plasmid encoding Renilla

A

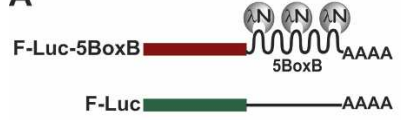

B Luciferase activity

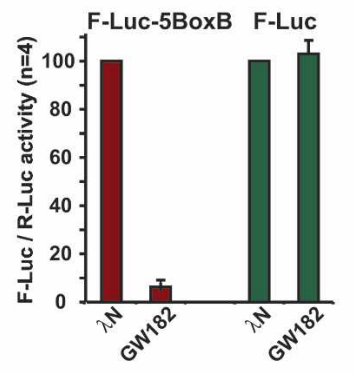

C Northern blot

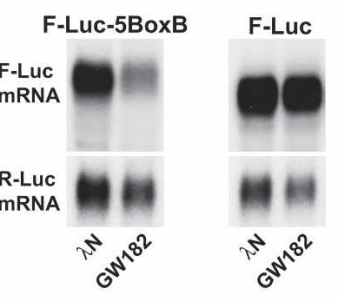

$\mathbf{F}$
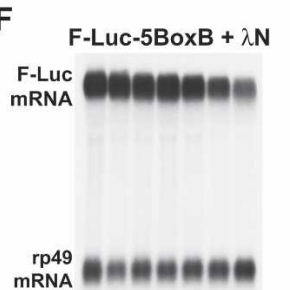

time $0,5 \wedge^{5} 3^{\circ} 6^{\circ} \wedge^{\circ} 3^{\circ}$

D mRNA levels

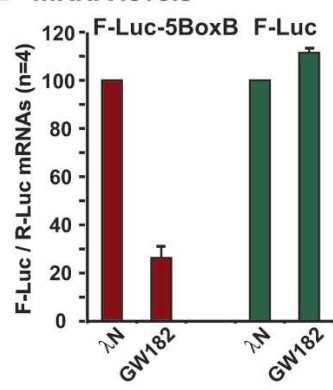

E Net effect on protein expression

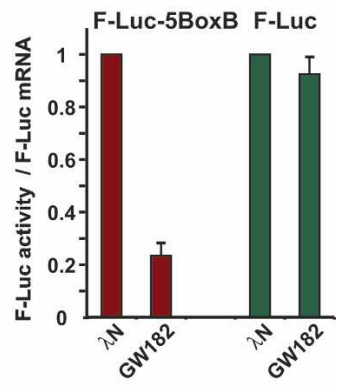

G

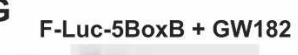

F-Luc
mRNA

RNA

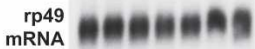

time $0,55^{5} 3^{\circ} 6^{\circ} 8_{8} 0_{3}^{\circ}$

$\mathrm{H}$

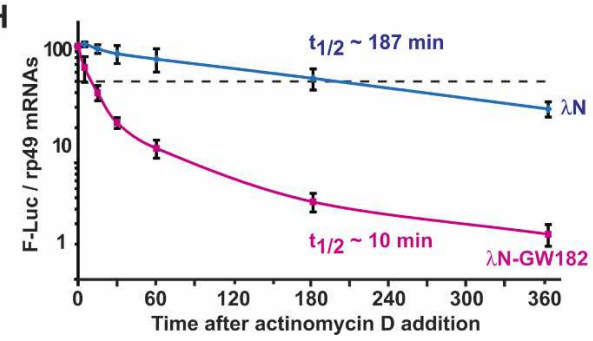


luciferase (R-Luc) as a transfection control. In the experiments described below, the firefly luciferase activity was normalized to that of Renilla to compensate for possible differences in transfection efficiencies.

We observed a 16-fold reduction in firefly luciferase expression relative to that in cells expressing the $\lambda \mathrm{N}$ peptide alone when $\lambda \mathrm{N}$-GW182 was tethered to the reporter transcript (Fig. 4B). The inhibitory effect of $\lambda \mathrm{N}$ GW182 on the expression of the F-Luc-5BoxB reporter was observed even when the amount of plasmid expressing $\lambda$ N-GW182 was reduced 200-fold (data not shown), suggesting that GW182 is a potent silencer of gene expression. Importantly, under these conditions other Pbody components fused to the $\lambda \mathrm{N}$-peptide showed no activity even if their expression levels were comparable to or higher than those of GW182 (e.g., $\lambda \mathrm{N}-\mathrm{Ge}-1, \lambda \mathrm{N}$ Staufen, $\lambda \mathrm{N}-\mathrm{DCP} 1, \lambda \mathrm{N}-\mathrm{Me} 31 \mathrm{~B})$, indicating that the silencing activity of GW182 is specific and not a generic property of P-body components. Moreover, the inhibitory effect of $\lambda \mathrm{N}$-GW182 affects only tethered mRNA, while a firefly luciferase reporter lacking the 5BoxBbinding sites is unaffected by overexpressed protein (FLuc) (Fig. 4A,B).

To determine whether GW182 silences firefly luciferase expression by inhibiting translation directly or indirectly by reducing mRNA levels, we analyzed the steadystate levels of the F-Luc-5BoxB mRNA by Northern blot and normalized them to the levels of the control R-Luc mRNA. Expression of the $\lambda \mathrm{N}-\mathrm{GW} 182$ fusion caused a marked reduction in the levels of the reporter mRNA (fourfold relative to the levels detected in cells expressing the $\lambda \mathrm{N}$-peptide alone) (Fig. $4 \mathrm{C}, \mathrm{D}$ ). We saw no effect on transcripts lacking the tethering sites (Fig. 4C,D).

The decrease in mRNA levels observed for the tethered mRNA does not, however, fully account for the strong reduction in firefly luciferase activity (Fig. 4, B vs. D). Indeed, after normalizing firefly luciferase activity to the corresponding mRNA levels, we saw that bound $\lambda \mathrm{N}$ GW182 leads to a net fourfold reduction in protein expression (Fig. 4E). These results suggest that GW182 silences expression of bound transcripts by two mechanisms: one involving inhibition of protein expression, and one involving a reduction of mRNA levels.

\section{GW182 increases the degradation rate} of bound $m R N A s$

We next asked whether the reduction of reporter mRNA levels caused by tethering of $\lambda$ N-GW182 was a consequence of increased mRNA degradation. To this end, the levels of F-Luc-5BoxB mRNA were analyzed over time after inhibition of transcription by actinomycin $\mathrm{D}$ and normalized to those of the long-lived (half-life $>8 \mathrm{~h}$ ) endogenous rp49 mRNA (which encodes ribosomal protein L32).

In cells expressing the $\lambda \mathrm{N}$-peptide alone, the half-life of F-Luc-5BoxB mRNA was $\sim 3 \mathrm{~h}$, while in cells expressing $\lambda \mathrm{N}$-GW182, the half-life of this mRNA was $\sim 10 \mathrm{~min}$ (Fig. 4F-H). mRNAs bound to $\lambda \mathrm{N}-\mathrm{GW} 182$ had biphasic decay kinetics, suggesting the existence of a heterogeneous pool of mRNAs (e.g., bound to GW182 with dif- ferent stoichiometries) undergoing different rates of degradation.

The half-lives of mRNAs lacking the tethering sites (F-Luc and the transfection control R-Luc mRNA) were not affected in cells expressing $\lambda \mathrm{N}$-GW182 (Supplementary Fig. S2; data not shown). Thus, tethering of GW182 causes a reduction of the steady-state levels of bound mRNAs by increasing their degradation rate. We conclude that the recruitment of GW182 (as mimicked in the tethering assay) marks bound mRNAs for decay.

\section{GW182 promotes mRNA deadenylation by recruiting the CCR4:NOT complex}

In cells expressing GW182, the F-Luc-5BoxB transcripts accumulating $15-30$ min after addition of actinomycin D were slightly shorter than transcripts isolated at time 0 (Fig. 4G). This increase in mobility suggests that GW182-mediated decay involves deadenylation. We therefore tested whether transcripts accumulating in cells expressing GW182 were polyadenylated. If this were the case, we would expect these transcripts to change their electrophoretic mobility upon removal of the poly(A)-tail by oligo(dT)-targeted ribonuclease $\mathrm{H}$ (RNase H) cleavage. When RNA samples isolated at time 0 were analyzed, both the F-Luc-5BoxB and the rp49 mRNAs changed mobility following removal of the poly(A)-tail, indicating that these mRNAs were polyadenylated (Fig. 5A, lanes 1,2). In contrast, for RNA samples isolated 15 min after the transcription block, the mobility of the luciferase reporter did not change after RNase $\mathrm{H}$ treatment in the presence of oligo(dT), while that of the rp49 mRNA did (Fig. 5A, lanes 3,4). These results indicate that GW182-mediated decay does involve deadenylation.

There are two major deadenylase complexes in Drosophila: the PAN2:PAN3 and the CCR4:NOT complexes (Temme et al. 2004). To investigate which of these complexes is involved in deadenylating mRNAs bound to GW182, we performed the tethering assay in cells depleted of PAN2, PAN3, or of two components of the CCR4:NOT complex (CAF1 and NOT1), whose depletion has been shown to affect deadenylation in S2 cells (Temme et al. 2004). In cells depleted of NOT1 (but not of PAN2:PAN3), the levels of the F-Luc-5BoxB mRNA bound to $\lambda$ N-GW182 were partially restored (Fig. $5 \mathrm{~B}, \mathrm{C})$, suggesting that GW182 requires the CCR4:NOT complex to degrade bound mRNAs efficiently. Depletion of CAF1, which leads to a simultaneous codepletion of CCR4 (Temme et al. 2004), also restored F-Luc-5BoxB mRNA levels, but to a lesser extent than the depletion of NOT1 (Fig. 5B,C). No restoration was observed in cells depleted of additional components of the CCR4:NOT complex (data not shown), suggesting that in S2 cells inactivation of this complex is most efficiently achieved by depletion of CAF1 or NOT1, as reported by Temme et al. (2004). Interestingly, the restoration of mRNA levels resulted in only a slight increase of firefly luciferase activity, indicating that the F-Luc-5BoxB mRNA was still translationally repressed (Fig. 5B). 
Figure 5. GW182 triggers decay by promoting deadenylation and decapping. (A) RNA samples shown in Figure 4G (lanes corresponding to time 0 and $15 \mathrm{~min}$ ) were treated with RNase $\mathrm{H}$ in the absence or presence of oligo(dT) and analyzed by Northern blot. (B) S2 cells depleted of GFP, CAF1, NOT1, or DCP1:DCP2 were transfected with plasmids for the $\lambda \mathrm{N}$-tethering assay as described in Figure 4. F-Luc activities and mRNA levels were quantitated in three independent experiments, normalized to that of the Renilla control, and set to 100 in cells expressing the $\lambda \mathrm{N}$-peptide alone for each knockdown (black bars). (C) Northern blot of representative RNA samples corresponding to $B$. (D) RNA samples shown in $C$ and corresponding to the DCP1 +2 knockdowns were treated with RNase $\mathrm{H}$ in the absence or presence of oligo(dT) and analyzed by Northern blot. (E) S2 cells were transfected with plasmids expressing miRNA reporters (Nerfin, Vha68-1, or CG10011), plasmids expressing miR-9b or miR-12 (gray bars), or the corresponding empty vector (black bars), as indicated. R-Luc served as a transfection control. Firefly luciferase activity and the corresponding mRNA levels were measured and normalized to those of the Renilla control. Normalized firefly luciferase activities and mRNA levels in cells transfected with the empty vector (black bars) were set to $100 \%$. Error bars represent standard deviations from at least three independent experiments. $(F)$ Northern blot of representative samples shown in $E$.
A

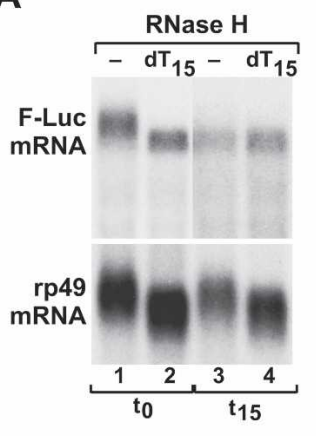

B

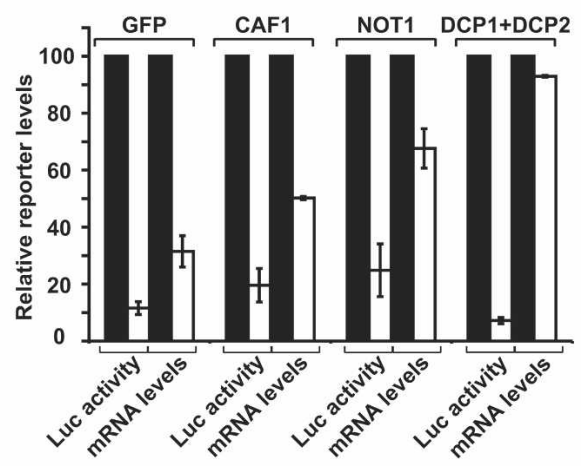

C

knock-

downs

F-Luc mRNA

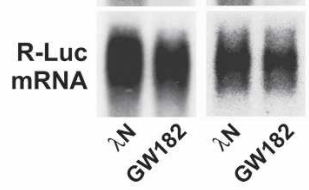

E

empty $\square+$ miRNA

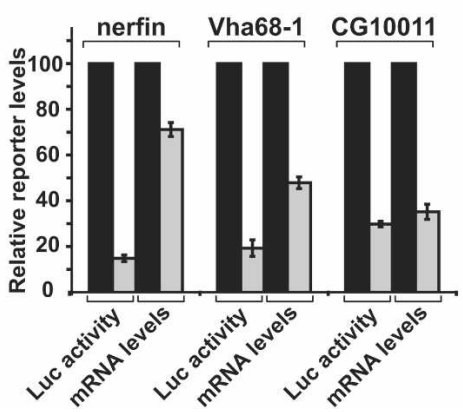

D

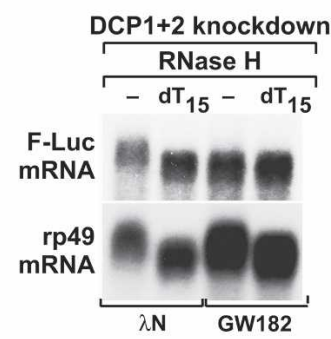

$\mathbf{F}$

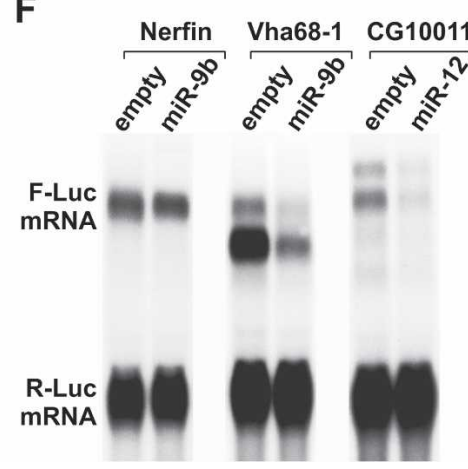

mRNA decay by GW182 requires the DCP1:DCP2 decapping complex

Given the role of the decapping DCP1:DCP2 complex in the miRNA pathway (Rehwinkel et al. 2005), we tested whether mRNA degradation triggered by GW182 also required decapping. To this end, we performed the $\lambda \mathrm{N}$ tethering assay in cells depleted of the DCP1:DCP2 decapping complex. Codepletion of DCP1 and DCP2 did not relieve the inhibition of luciferase expression caused by tethered GW182 (Fig. 5B).

When mRNA levels where analyzed, we observed that depletion of the decapping complex restored the levels of the reporter mRNA up to the levels observed in depleted cells expressing the $\lambda \mathrm{N}$-peptide alone (Fig. 5B,C). Nevertheless, in cells expressing $\lambda N$-GW182, the accumulated transcripts were shorter and comigrate with the deadenylated form (Fig. 5C,D). This finding provides a possible explanation for the lack of restoration of luciferase activity despite restoration of mRNA levels, since less efficient translation of deadenylated transcripts is to be expected. Altogether, our results indicate that GW182- mediated mRNA decay involves deadenylation by the CCR4:NOT complex and decapping by the DCP1:DCP2 complex.

mRNA decay triggered by miRNAs occurs via a deadenylation and decapping mechanism requiring GW182

To assess whether mRNA degradation by miRNAs is dependent on GW182 and involves the CCR4:NOT and DCP1:DCP2 complexes, we made use of firefly luciferase reporters described before (Rehwinkel et al. 2005). In these reporters the coding region of firefly luciferase is flanked by the 3' UTRs of the Drosophila gene CG10011 (F-Luc-CG10011), regulated by miR-12, and of Nerfin or Vha68-1 (F-Luc-Nerfin, F-Luc-Vha68-1), regulated by miR-9b. Expression of F-Luc-CG10011 is strongly reduced by coexpression of miR-12, while expression of F-Luc-Nerfin or F-Luc-Vha68-1 is inhibited by coexpression of miR-9b (Fig. 5E; Rehwinkel et al. 2005, 2006).

Analysis of the steady-state levels of the reporters in- 
dicated that miRNAs trigger reductions in mRNA levels to different extents (Fig. 5E,F). When we compared the luciferase activity to the mRNA levels we saw that miR$9 \mathrm{~b}$ led mainly to a net decrease in protein expression from the Nerfin reporter. In contrast, silencing of F-LucCG10011 by miR-12 can be attributed entirely to the reduction of mRNA levels (Fig. 5E,F). The Vha68-1 reporter displayed an intermediate behavior with effects on both protein expression and mRNA levels (Fig. 5E,F). Thus, like GW182, miRNAs silence gene expression by inhibiting protein expression and/or by reducing mRNA levels.
We next transfected the miRNA reporters together with the cognate miRNAs into cells depleted of GW182, CAF1, NOT1, or the DCP1:DCP2 decapping complex. As a control, cells depleted of AGO1 or GFP were also transfected. For the three reporters, depletion of AGO1 or GW182 relieved the inhibition of luciferase expression caused by the miRNAs (Figs. 6A,B, 7A). In agreement with this, reporter mRNA levels (in particular F-Luc-Vha68-1 and F-Luc-CG10011 mRNAs) were restored, indicating that mRNA decay triggered by miRNAs requires both AGO1 and GW182 (Figs. 6A-D, 7A). Notably, endogenous Vha68-1 and CG10011 mRNAs are

\section{A Nerfin}

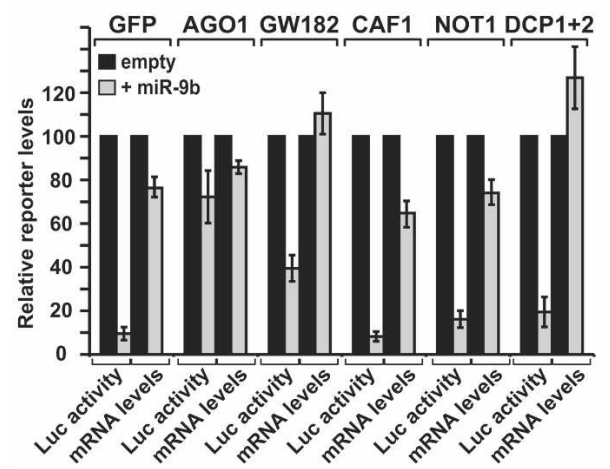

C Nerfin

knock-
downs GFP AGO1 GW182 CAF1 NOT1 DCP1+2
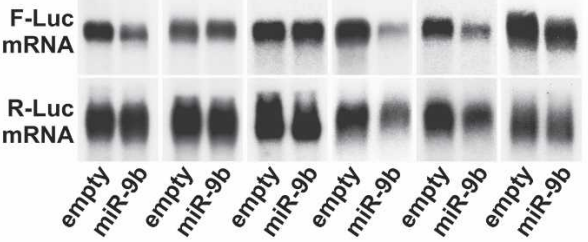

E Nerfin

DCP1+2 knockdown

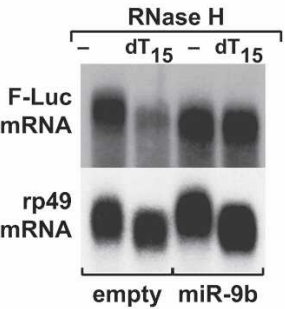

F CG10011
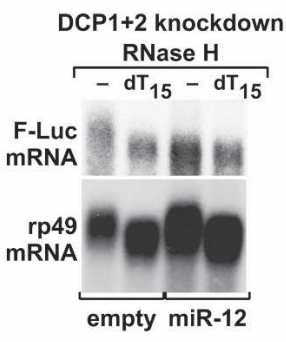

\section{B CG10011}

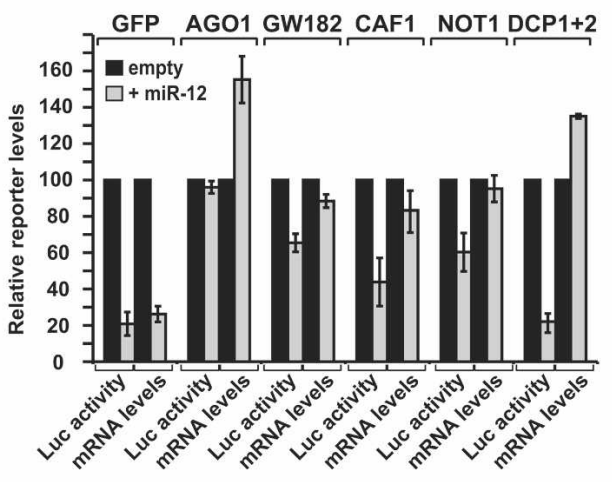

\section{CG10011}

knock-

downs GFP AGO1 GW182 CAF1 NOT1 DCP1+2
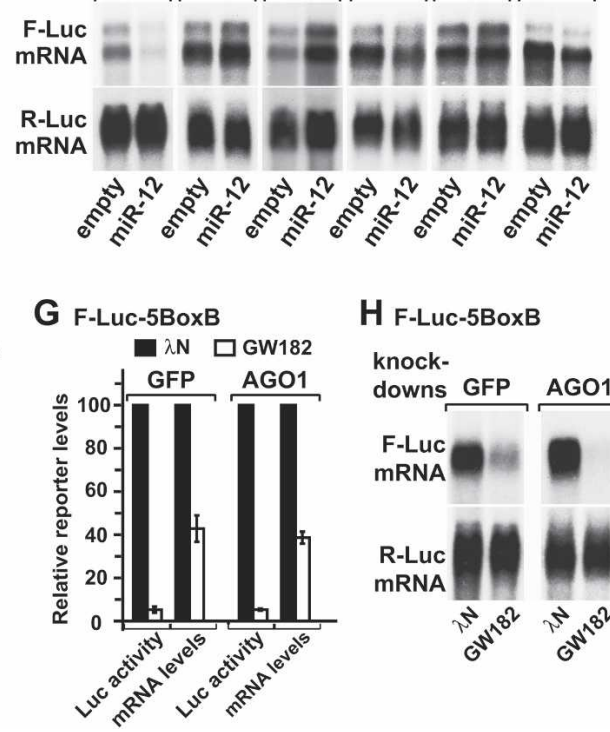

Figure 6. mRNA decay triggered by miRNAs occurs by a deadenylation and decapping mechanism requiring GW182. (A,B) S2 cells depleted of GFP, AGO1, GW182, CAF1, NOT1, or the DCP1:DCP2 decapping complex were transfected with plasmids expressing the miRNA reporters described in Figure 5E. Firefly luciferase activity and the corresponding mRNA levels were measured and normalized to those of the Renilla control. Normalized firefly luciferase activities and mRNA levels in cells transfected with the empty vector (black bars) were set to $100 \%$ for each knockdown. Error bars represent standard deviations from three independent experiments. (C,D) Northern blot of representative samples shown in $A$ and $B .(E, F)$ RNA samples shown in $C$ and $D$, lanes corresponding to the DCP1 +2 knockdown, were treated with RNase $\mathrm{H}$ in the absence or presence of oligo(dT) and analyzed by Northern blot. $(G, H) \mathrm{S} 2$ cells treated with dsRNAs targeting GFP or AGO1 were transfected with plasmids for the $\lambda \mathrm{N}$-tethering assay. Firefly luciferase activity and the corresponding mRNA levels were measured and normalized to those of the Renilla control as described in Figure 5. $(H)$ Northern blot of representative mRNA samples shown in $G$. 
also up-regulated in AGO1- or GW182-depleted cells (Figs. 1E, 7B; Nerfin mRNA is not expressed in S2 cells).

In NOT1-depleted cells we observed a full restoration of mRNA levels for the reporters for which the cognate miRNA triggered degradation (i.e., CG10011 and Vha681) (Figs. 6B,D, 7A). For these reporters we also observed a partial restoration of luciferase expression (Figs. 6B, 7A). Restoration was also observed, but was less pronounced, in cells depleted of CAF1 (Figs. 6B,D, 7A). In contrast, silencing of the Nerfin reporter, which occurs mainly at the translational level, was not relieved in cells depleted of CAF1 or NOT1 (Fig. 6A,C).

Depletion of the DCP1:DCP2 decapping complex led to a complete restoration of mRNA levels for the three reporters (Figs. 6A-D, 7A). The accumulated transcripts were deadenylated, as judged by the unchanged mobility in the presence of oligo(dT) and RNase H (Fig. 6E,F). Remarkably, although the levels of the Nerfin reporter are not strongly affected by miR-9b, the mRNA is deadenylated in cells depleted of DCP1:DCP2 (Fig. 6E). The accumulation of deadenylated transcripts in cells depleted of the decapping enzymes appears to be specific to mRNAs undergoing GW182- or miRNA-mediated decay, because mRNAs that are not regulated by miRNAs are still polyadenylated in these cells (e.g., rp49 mRNA, R-Luc mRNA, and the F-Luc miRNA reporters in the absence of a cognate miRNA) (Figs. 5D, 6C-F, 7C).

The observation that transcripts accumulating in DCP1:DCP2-depleted cells are deadenylated provides an explanation for the lack or partial restoration of luciferase activity. In contrast, the partial restoration of luciferase activity for the CG10011 and Vha68-1 reporters, together with the lack of restoration for the Nerfin reporter in cells depleted of CAF1 or NOT1, suggests that translational repression by miRNAs is not relieved in these cells. We conclude that gene silencing by miRNAs involves translational repression and/or mRNA deadenylation and decapping and both require GW182.

To investigate whether the regulation of the F-Luc re-

Figure 7. Endogenous miRNA targets are degraded by deadenylation and decapping. (A) S2 cells depleted of GFP, AGO1, GW182, CAF1, NOT1, or the DCP1:DCP2 decapping complex were transfected with the F-Luc-Vha68-1 reporter. Firefly luciferase activity and the corresponding mRNA levels were measured and analyzed as described in Figure 6, A and B. (B) Expression levels of endogenous Vha68-1 mRNA in cells depleted of AGO1, GW182, CAF1, or DCP1:DCP2. The signals from the Northern blot were normalized to rp49 mRNA (not shown). These values are compared with the values measured by microarray. Values are given as fold changes relative to the values obtained cells treated with GFP dsRNA. (n.d.) Not determined. $(C)$ RNA samples shown in $B$, lanes corresponding to the control and the DCP1 +2 knockdown, were treated with RNase $\mathrm{H}$ in the absence or presence of oligo(dT) and analyzed by Northern blot. $(D, E)$ The decay of Vha68-1 and Axs mRNAs was monitored in depleted cells following inhibition of transcription by actinomycin D. The levels of the Vha68-1 and Axs mRNAs were normalized to rp49 mRNA and plotted against time (not shown for Vha68-1 mRNA). mRNA half-lives $\left(t_{1 / 2}\right)$ calculated from the decay curves are indicated. porters by coexpressed miRNAs reflects the genuine miRNA pathway, we performed a similar analysis for endogenous Vha68-1 and Axs mRNAs, which are predicted targets of miR-9b and miR-285, respectively. Vha68-1 and Axs mRNA levels were up-regulated in cells depleted of AGO1 or GW182, in agreement with the array data (Figs. 1E,F, 7B). These mRNAs were also up-regulated in cells depleted of CAF1, NOT1, or of DCP1:DCP2 (Fig. 7B; data not shown). As observed for
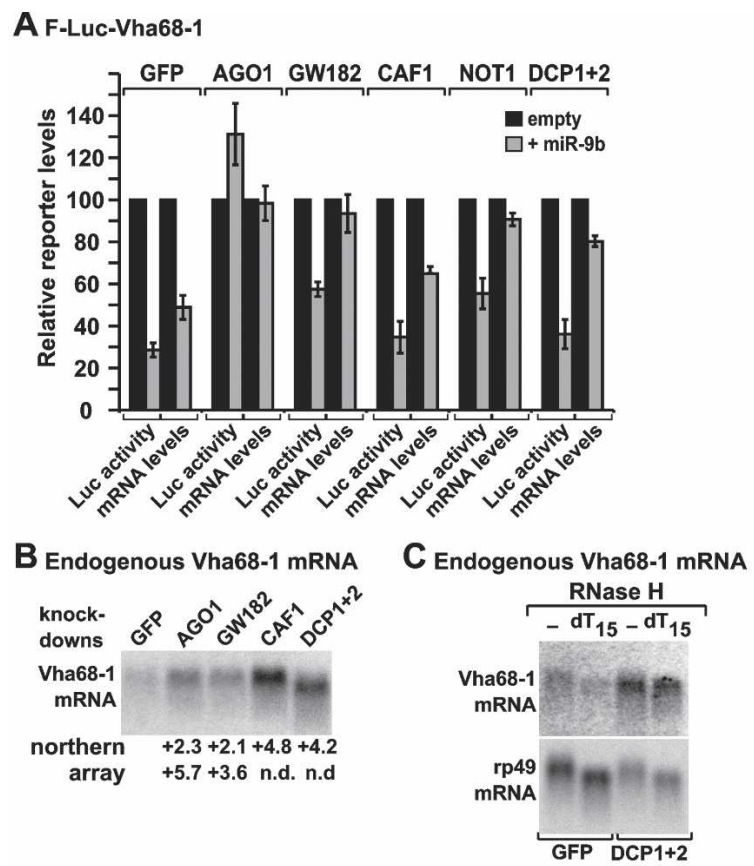

D
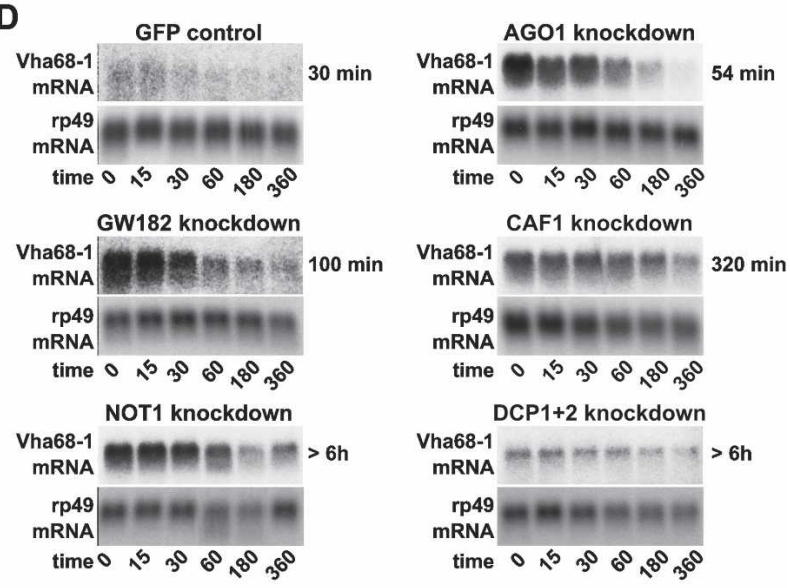

E Endogenous Axs mRNA

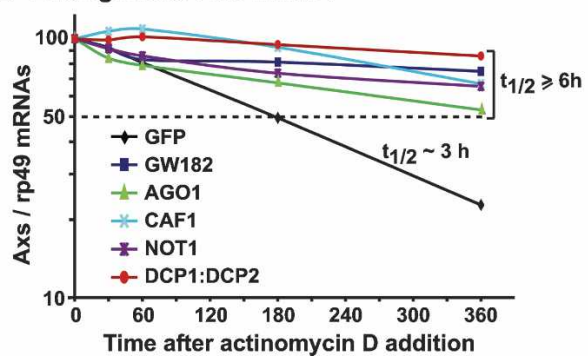


the F-Luc reporters, Vha68-1 transcripts accumulating in DCP1:DCP2-depleted cells were deadenylated (Fig. 7B,C). The increased levels of Vha68-1 and Axs mRNAs in cells depleted of AGO1, GW182, CAF1, NOT1, or DCP1:DCP2 were all accompanied by a corresponding increase in the half-life of the mRNA (Fig. 7D,E).

The results obtained by analyzing endogenous miRNA targets are qualitatively similar to those obtained with the F-Luc reporters. However, in contrast to the reporters, we observed a stronger stabilization of Vha68-1 mRNA in cells depleted of CAF1, NOT1, or DCP1:DCP2 relative to the stabilization observed in cells depleted of AGO1 or GW182. The simplest explanation for these differences is that only a fraction of endogenous Vha68-1 mRNA is degraded by the miRNA pathway. In agreement with this possibility, miR-9b is expressed at very low levels in S2 cells (Rehwinkel et al. 2006). Thus, for this and probably other endogenous miRNA targets, depletion of decapping enzymes and of components of the CCR4:NOT complex is likely to prevent both normal decay and decay by miRNAs, giving raise to a stronger stabilization of the mRNA than that observed when only the miRNA pathway is inhibited.

\section{Tethering of GW182 bypasses the requirement for $A G O 1$}

The results described above, together with previous studies, show that GW182 is required for silencing of luciferase expression mediated by miRNAs (Fig. 6; Jakymiw et al. 2005; Liu et al. 2005b; Meister et al. 2005; Rehwinkel et al. 2005). It was therefore of interest to investigate whether silencing triggered by GW182 required AGO1. To address this question, we performed the tethering assays in cells depleted of AGO1. The inhibition of luciferase expression and the reduction of mRNA levels caused by tethering of GW182 were not relieved in cells depleted of AGO1 (Fig. 6G,H). This lack of restoration in AGO1-depleted cells cannot be explained by inefficient depletion, because silencing of the miRNA reporters was relieved in these cells (Fig. 6A-D). Thus, depletion of AGO1 prevented miRNA-mediated silencing but not silencing elicited by tethering of GW182. We conclude that tethering of GW182 bypasses the requirement for AGO1. Although the possibility that AGO2 substitutes for AGO1 when the latter is depleted cannot be ruled out, we consider it unlikely as AGO2 did not interact detectably with GW182.

\section{Discussion}

The results presented here together with previous studies (Ding et al. 2005; Jakymiw et al. 2005; Liu et al. 2005b; Meister et al. 2005; Rehwinkel et al. 2005) indicate that GW182 is a genuine component of RNA silencing pathways, associating with the Argonaute proteins and with components of the mRNA decay machinery and, providing a molecular link between RNA silencing and mRNA degradation.
Drosophila GW182 is a component

of the miRNA pathway

Depletion of GW182 or AGO1 from Drosophila cells leads to correlated changes in mRNA expression profiles, indicating that these proteins act in the same pathway. Transcripts commonly up-regulated by AGO1 and GW182 are enriched in predicted and validated miRNA targets. These results, together with the observation that GW182 associates with AGO1, identify GW182 as a component of the miRNA pathway.

GW182 belongs to a protein family with GW repeats, a central UBA domain, and a C-terminal RRM. Multiple sequence alignment of all proteins possessing these domains revealed that there are three paralogs (TNRC6A/ GW182, TNRC6B, and TNRC6C) in vertebrates, a single ortholog in insects, and no orthologs in worms or fungi. At present, it is unclear whether the vertebrate paralogs have redundant functions, but both GW182 and TNRC6B have been shown to associate with human AGO1 and AGO2 (Jakymiw et al. 2005; Liu et al. 2005b; Meister et al. 2005).

In Drosophila, GW182 interacts with AGO1 in vivo and in vitro. We could not detect a stable interaction with AGO2 under the same conditions, suggesting that AGO2 may act independently of GW182. This is consistent with the observation that depletion of GW182 does not affect siRNA-guided mRNA cleavage or RNAi, which is mediated exclusively by AGO2 in Drosophila (Okamura et al. 2004; Miyoshi et al. 2005; Rehwinkel et al. 2005). Nevertheless, since AGO2 also regulates the expression levels of a subset of miRNA targets (Rehwinkel et al. 2006), the lack of interaction with GW182 raises the question of whether this regulation occurs by a similar or different mechanism from that mediated by AGO1. Further studies are needed to elucidate the mechanism by which Drosophila AGO2 regulates the expression of a subset of miRNA targets.

\section{GW182 has a conserved modular domain organization}

The N-terminal GW repeat region of GW182 encompasses two highly conserved motifs (I and II) and is expanded in vertebrates. This region is shorter in insects and bears similarity to the GW-like regions in the $C$. elegans protein AIN-1, involved in the miRNA pathway (Ding et al. 2005). However, AIN-1 does not contain UBA, Q-rich, or RRM domains. This lack of common domain architecture suggests that AIN-1 represents a functional analog. Nevertheless, the observation that $C$. elegans AIN-1 also localizes to P-bodies and interacts with AGO1 (i.e., worm ALG-1), and the finding that the N-terminal GW repeats of Drosophila GW182 interact with the PIWI domain of AGO1, suggest a conserved role for these repeats in mediating the interaction with Argonaute proteins. It would be of interest to determine the molecular basis of the specific interaction between the $\mathrm{N}$-terminal GW repeats of GW182 and the PIWI domain of AGOs, and whether this interaction affects the catalytical activity of the domain. 
Apart from the interaction with AGO1, the N-terminal repeats and the UBA and Q-rich domains contribute to the localization of GW182 in P-bodies, which is in turn required for P-body integrity (Eystathioy et al. 2002, 2003). This suggests that GW182 may act as a molecular scaffold bringing together AGO1-containing RISCs and mRNA decay enzymes, possibly nucleating the assembly of P-bodies. Understanding the precise role of the various GW182 domains in the interaction with mRNA decay enzymes and AGO1 as well as in P-body integrity awaits further biochemical characterization.

\section{GW182 silences the expression of bound transcripts by inhibiting protein expression and promoting mRNA decay}

Tethering GW182 to a reporter transcript silences its expression, bypassing the requirement for AGO1. Silencing by GW182 occurs by two distinct mechanisms: repression of protein expression, and mRNA degradation. It remains to be elucidated how GW182 represses translation. We show that mRNA degradation by GW182 is inhibited in cells depleted of CAF1, NOT1, or the DCP1:DCP2 complex, indicating that GW182 promotes mRNA deadenylation and decapping. Thus, binding of GW182 appears to be a point of no return, which marks transcripts as targets for degradation.

More studies are needed to determine whether decapping triggered by GW182 requires prior deadenylation or whether these two events occur independently. The observation that mRNA levels are fully restored in cells depleted of DCP1:DCP2, suggests that deadenylation followed by 3 '-to-5' exonucleolytic degradation is unlikely to represent a major pathway by which these mRNAs are degraded. Future studies should also reveal the identity of the nuclease(s) acting downstream of the decapping enzymes.

miRNAs silence gene expression in a GW182-dependent manner

Previous studies indicate that miRNAs can reduce the levels of the targeted transcripts, and not just the expression of the translated protein (Bagga et al. 2005; Lim et al. 2005; Giraldez et al. 2006; Rehwinkel et al. 2006; Wu et al. 2006). Consistently, transcripts up-regulated in cells depleted of AGO1 or GW182 are enriched in predicted and validated miRNA targets (Rehwinkel et al. 2006; this study). In this paper we provide further evidence indicating that miRNAs silence gene expression by two mechanisms: one mechanism involving translational silencing, and one involving mRNA degradation. The contribution of these mechanisms to miRNA-mediated gene silencing appears to differ for each miRNA:target pair. Indeed, of the three reporters we have analyzed, Nerfin is silenced mainly at the translational level, silencing of the CG10011 reporter can be attributed to mRNA degradation, while Vha68-1 is regulated both at the translational and mRNA levels. Regardless of the extent of the contribution of these two mechanisms to silencing, both require AGO1 and GW182, because the levels of the mRNA reporter and luciferase activity are restored in cells depleted of any of these two proteins.

In contrast, although the levels of the mRNA reporter are restored in cells depleted of CAF1 or NOT1, translational repression is not fully relieved, indicating that deadenylation is required for mRNA decay, but not for translational silencing by miRNAs. In agreement with this, two reports published while this manuscript was in preparation have shown that miRNAs trigger accelerated deadenylation of their targets (Giraldez et al. 2006; Wu et al. 2006). Our study extends these observations further by demonstrating: First, deadenylation is mediated by the CCR4:NOT complex; second, decapping is also required for miRNA target degradation; finally, we show that both deadenylation and decapping triggered by miRNAs requires GW182.

Based on the results presented in this study and the observations that GW182 associates with AGO1 and is required for miRNA-mediated gene silencing (Ding et al. 2005; Jakymiw et al. 2005; Liu et al. 2005b; Meister et al. 2005; Rehwinkel et al. 2005), we propose the following model: AGO1-containing RISCs binds to mRNA targets by means of base-pairing interactions with miRNAs; AGO1 may then recruit GW182, which marks the transcripts as targets for decay via a deadenylation and decapping mechanism.

A question that remains open is whether miRNA-mediated translational repression is the cause of mRNA degradation or whether these represent two independent mechanism by which miRNAs silence gene expression as proposed by $\mathrm{Wu}$ et al. (2006). Indeed, changes in mRNA levels are not observed for all miRNA targets (Rehwinkel et al. 2006), suggesting that inhibition of translation is not always followed by mRNA decay. Conversely, depletion of CAF1 or NOT1 prevents mRNA decay but does not relieve translational silencing, suggesting that these two processes are independent.

An important finding is that miRNAs elicit degradation to different extents. One possible explanation is that the extent of degradation depends on the stability of the miRNA:mRNA duplexes. Also, the extent of degradation might depend on the particular set of proteins associated with a given target. For instance, some targets may assemble with a set of proteins that antagonize degradation. Finally, GW182 might interact only with a subset of AGO1-containing RISCs, as suggested for AIN-1 (Ding et al. 2005). A major challenge will be to identify the specific features of miRNA targets and/or RISC complexes that lead to regulation of gene expression at the level of translation or at the level of mRNA stability.

\section{Materials and methods}

RNAi and genome-wide expression analysis

RNAi was performed essentially as described by Rehwinkel et al. $(2004,2006)$. Total RNA was isolated from control and depleted cells using peqGOLD TriFast reagent (peqlab Biotech- 
nologie). To reduce potential variations in the preparation of the RNA, two RNA preparations were isolated from a single knockdown experiment. Pools of two RNA preparations from a single knockdown are referred to as RNA samples.

High-density oligonucleotide microarrays (Affymetrix-Drosophila array 2) covering $>18,500$ transcripts from Drosophila were used. The microarray results have been submitted to the ArrayExpress database at EBI under accession number E-MEXP487. Standard Affymetrix protocols were used for preparation of biotinylated targets, hybridization, and data acquisition. Control parameters were within recommended limits. All experiments were normalized using an intensity-dependent normalization scheme (Lowess). When two independent total RNA samples were compared, all spots after intensity-dependent normalization had an average ratio of $1.08 \pm 0.54$. We therefore apply a twofold cutoff to identify regulated transcripts.

\section{Sequence analysis}

Sequence similarity searches were performed using PSI-BLAST (Altschul et al. 1997), with Drosophila and human GW182 as input sequences. A group of putatively related sequences was defined on the basis of the presence of GW repeats and a Cterminal RRM. The relationship between these proteins was confirmed by using each of the sequences as an input for PSIBLAST and retrieving all the other members of the group.

\section{Transfection of S2 cells, RNA analysis, and luciferase assays}

Plasmids allowing the expression of miRNA primary transcripts, miRNA reporters, and the F-Luc-5BoxB plasmid are described by Rehwinkel et al. (2005) and Stark et al. (2005). For the expression of $\lambda \mathrm{N}$-HA-peptide fusions, cDNAs encoding fulllength GW182 or protein domains were amplified with primers containing appropriate restriction sites, using a $(\mathrm{dT})_{15}$-primed S2 cDNA library as a template. The amplified cDNAs were cloned into a vector allowing the expression of $\lambda \mathrm{N}$-HA-peptide fusions (pAc5.1- $\lambda \mathrm{N}-\mathrm{HA})$.

Transfections were performed in six- or 24-well dishes using Effectene transfection reagent (Qiagen). For the $\lambda \mathrm{N}$-tethering assay, the following plasmids were cotransfected: $0.15 \mu \mathrm{g}$ reporter plasmid (F-Luc-5BoxB or F-Luc), $0.4 \mu \mathrm{g}$ pAc5.1-R-Luc as transfection control, and $5 \mathrm{ng}$ to $1 \mu \mathrm{g}$ pAc5.1 $\lambda \mathrm{N}-\mathrm{HA}$ construct for the expression of $\lambda \mathrm{N}-\mathrm{HA}$ fusions. To test miRNA function, the following plasmids were cotransfected: $0.1 \mu \mathrm{g}$ of firefly luciferase reporter plasmid, $0.3 \mu \mathrm{g}$ pAc5.1-R-Luc as transfection control, and $1 \mu \mathrm{g}$ of pAc5.1 plasmid without insert (empty vector) or expressing miRNA primary transcripts.

Three days after transfection, firefly and Renilla luciferase activities were measured using the Dual-Luciferase reporter assay system (Promega), and total RNA was isolated using TriFast (peqlab biotechnologies). RNA samples were analyzed as described by Rehwinkel et al. (2004).

For the measurement of mRNA half-lives, transfected cells were treated with actinomycin D ( $5 \mu \mathrm{g} / \mathrm{mL}$ final concentration) $3 \mathrm{~d}$ after transfection, and harvested at the time points indicated. RNase $\mathrm{H}$ (USB) digestion using a $(\mathrm{dT})_{15}$ oligonucleotide was performed according to the manufacturer's instructions.

\section{Fluorescence microscopy}

S2 cells were allowed 15 min to adhere to poly-D-Lysine-coated coverslips, fixed with $3.7 \%$ paraformaldehyde in PBS for $10 \mathrm{~min}$, and permeabilized for 15 min with PBS containing $0.1 \%$ Triton X-100. HA-tagged proteins were detected with monoclonal antiHA antibody (Covance Research Products) diluted 1:1000 in
PBS containing $1 \%$ bovine serum albumin (BSA). TRITCcoupled goat secondary antibody (Molecular Probes) was used in a dilution of 1:250. For detection of endogenous GW182, S2 cells were fixed with $90 \%$ Methanol and 10\% formaldehyde, permeabilized with $0.1 \%$ Triton X-100 in PBS, and stained with affinity-purified anti-GW182 antibody diluted 1:400 in PBS containing $1 \%$ BSA. DNA was stained with Hoechst 33342 (Molecular Probes). Cells were mounted using Fluoromount-G (Southern Biotechnology Associates, Inc.). Images were acquired using a Zeiss LSM510 META confocal microscope.

Western blots, coimmunoprecipitations, and GST pull-downs

Western blotting was performed with polyclonal anti-HA antibodies (Sigma) or anti-GW182 antibodies, using the CDP-Star chemiluminescent immunoblot system (PE Biosystems). Drosophila AGO1 was detected using a rabbit polyclonal antibody (AbCam). The primary antibodies were diluted 1:1000 and detected with alkaline-phosphatase-coupled secondary antibodies (Western-Star kit from Tropix).

For coimmunoprecipitations, S2 cells were collected $3 \mathrm{~d}$ after transfection, washed with PBS, and lysed for $15 \mathrm{~min}$ on ice in NET buffer (50 mM Tris at pH 7.4, $150 \mathrm{mM} \mathrm{NaCl}, 1$ mM EDTA, $0.1 \% \mathrm{NP} 40$ ). Cells were spun at $13,000 \mathrm{rpm}$ for $15 \mathrm{~min}$ at $4^{\circ} \mathrm{C}$. Anti-HA antibodies (Covance) were added to the supernatants $\left(2.5 \mu \mathrm{L} / 2 \times 10^{6}\right.$ cells $)$. After $1 \mathrm{~h}$ at $4^{\circ} \mathrm{C}, 25 \mu \mathrm{L}$ of Protein Gagarose (Roche) were added and the mixtures were rotated for 1 $\mathrm{h}$ at $4^{\circ} \mathrm{C}$. Beads were washed three times with NET buffer and once with PBS. Bound proteins were eluted with protein sample buffer.

For synthesis of $\left[{ }^{35} \mathrm{~S}\right]$-labeled in vitro translated proteins, the combined in vitro transcription/translation (TnT) kit from Promega was used following the manufacturer's instructions. GST and GST-GW182 (1-592) were expressed in Escherichia coli BL21(DE3).

\section{Acknowledgments}

We are grateful to members of the Gene Core Facility at EMBL for performing the array hybridizations, J. Brennecke and S.M. Cohen for the Nerfin reporter, and S. Danes and D.J. Thomas for comments on the manuscript. This study was supported by the European Molecular Biology Organization (EMBO) and the Human Frontier Science Program Organization (HFSPO).

\section{References}

Altschul, S.F., Madden, T.L., Schaffer, A.A., Zhang, J., Zhang, Z., Miller, W., and Lipman, D.J. 1997. Gapped BLAST and PSI-BLAST: A new generation of protein database search programs. Nucleic Acids Res. 25: 3389-3402.

Bagga, S., Bracht, J., Hunter, S., Massirer, K., Holtz, J., Eachus, R., and Pasquinelli, A.E. 2005. Regulation by let-7 and lin-4 miRNAs results in target mRNA degradation. Cell 122: 553 563.

Bartel, D.P. 2004. MicroRNAs: Genomics, biogenesis, mechanism, and function. Cell 116: 281-297.

Ding, L., Spencer, A., Morita, K., and Han, M. 2005. The developmental timing regulator AIN-1 interacts with miRISCs and may target the argonaute protein ALG-1 to cytoplasmic $P$ bodies in C. elegans. Mol. Cell 19: 437-447.

Eystathioy, T., Chan, E.K., Tenenbaum, S.A., Keene, J.D., Griffith, K., and Fritzler, M.J. 2002. A phosphorylated cytoplasmic autoantigen, GW182, associates with a unique population of human mRNAs within novel cytoplasmic speckles. 
Mol. Biol. Cell 13: 1338-1351.

Eystathioy, T., Jakymiw, A., Chan, E.K., Seraphin, B., Cougot, N., and Fritzler, M.J. 2003. The GW182 protein colocalizes with mRNA degradation associated proteins hDcpl and hLSm4 in cytoplasmic GW-bodies. RNA 9: 1171-1173.

Filipowicz, W. 2005. RNAi: The nuts and bolts of the RISC machine. Cell 122: 17-20.

Giraldez, A.J., Mishima, Y., Rihel, J., Grocock, R.J., Van Dongen, S., Inoue, K., Enright, A.J., and Schier, A.F. 2006. Zebrafish MiR-430 promotes deadenylation and clearance of maternal mRNAs. Science 312: 75-79.

Humphreys, D.T., Westman, B.J., Martin, D.I., and Preiss, T. 2005. MicroRNAs control translation initiation by inhibiting eukaryotic initiation factor 4E/cap and poly(A) tail function. Proc. Natl. Acad. Sci. 102: 16961-16966.

Jakymiw, A., Lian, S., Eystathioy, T., Li, S., Satoh, M., Hamel, J.C., Fritzler, M.J., and Chan, E.K. 2005. Disruption of GW bodies impairs mammalian RNA interference. Nat. Cell Biol. 7: 1167-1174.

Jing, Q., Huang, S., Guth, S., Zarubin, T., Motoyama, A., Chen, J., Di Padova, F., Lin, S.C., Gram, H., and Han, J. 2005. Involvement of microRNA in AU-rich element-mediated mRNA instability. Cell 120: 623-634

Kim, J., Krichevsky, A., Grad, Y., Hayes, G.D., Kosik, K.S., Church, G.M., and Ruvkun, G. 2004. Identification of many microRNAs that copurify with polyribosomes in mammalian neurons. Proc. Natl. Acad. Sci. 101: 360-365.

Lim, L.P., Lau, N.C., Garrett-Engele, P., Grimson, A., Schelter, J.M., Castle, J., Bartel, D.P., Linsley, P.S., and Johnson, J.M. 2005. Microarray analysis shows that some microRNAs downregulate large numbers of target mRNAs. Nature 433: 769-773.

Liu, J., Valencia-Sanchez, M.A., Hannon, G.J., and Parker, R. 2005a. MicroRNA-dependent localization of targeted mRNAs to mammalian P-bodies. Nat. Cell Biol. 7: 719-723.

Liu, J., Rivas, F.V., Wohlschlegel, J., Yates, J.R., Parker, R., and Hannon, G.J. 2005b. A role for the P-body component GW182 in microRNA function. Nat. Cell Biol. 7: 1261-1266.

Meister, G., Landthaler, M., Peters, L., Chen, P.Y., Urlaub, H., Luhrmann, R., and Tuschl, T. 2005. Identification of novel argonaute-associated proteins. Curr. Biol. 15: 2149-2155.

Miyoshi, K., Tsukumo, H., Nagami, T., Siomi, H., and Siomi, M.C. 2005. Slicer function of Drosophila Argonautes and its involvement in RISC formation. Genes \& Dev. 19: 28372848.

Nelson, P.T., Hatzigeorgiou, A.G., and Mourelatos, Z. 2004 miRNP:mRNA association in polyribosomes in a human neuronal cell line. RNA 10: 387-394.

Okamura, K., Ishizuka, A., Siomi, H., and Siomi, M.C. 2004. Distinct roles for Argonaute proteins in small RNA-directed RNA cleavage pathways. Genes \& Dev. 18: 1655-1666.

Olsen, P.H. and Ambros, V. 1999. The lin-4 regulatory RNA controls developmental timing in Caenorhabditis elegans by blocking LIN-14 protein synthesis after the initiation of translation. Dev. Biol. 216: 671-680.

Petersen, C.P., Bordeleau, M.E., Pelletier, J., and Sharp, P.A. 2006. Short RNAs repress translation after initiation in mammalian cells. Mol. Cell 21: 533-542.

Pillai, R.S., Artus, C.G., and Filipowicz, W. 2004. Tethering of human Ago proteins to mRNA mimics the miRNA-mediated repression of protein synthesis. RNA 10: 1518-1525.

Pillai, R.S., Bhattacharyya, S.N., Artus, C.G., Zoller, T., Cougot, N., Basyuk, E., Bertrand, E., and Filipowicz, W. 2005. Inhibition of translational initiation by let-7 microRNA in human cells. Science 309: 1573-1576.
Rand, T.A., Ginalski, K., Grishin, N.V., and Wang, X. 2004 Biochemical identification of Argonaute 2 as the sole protein required for RNA-induced silencing complex activity. Proc. Natl. Acad. Sci. 101: 14385-14389.

Rehwinkel, J., Herold, A., Gari, K., Kocher, T., Rode, M., Ciccarelli, F.L., Wilm, M., and Izaurralde, E. 2004. Genomewide analysis of mRNAs regulated by the THO complex in Drosophila melanogaster. Nat. Struct. Mol. Biol. 11: 558566.

Rehwinkel, J., Behm-Ansmant, I., Gatfield, D., and Izaurralde, E. 2005. A crucial role for GW182 and the DCP1:DCP2 decapping complex in miRNA-mediated gene silencing. RNA 11: $1640-1647$.

Rehwinkel, J., Natalin, P., Stark, A., Brennecke, J., Cohen, S.M. and Izaurralde, E. 2006. Genome-wide analysis of mRNAs regulated by Drosha and Argonaute proteins in Drosophila. Mol. Cell. Biol. 26: 2965-2975.

Sen, G.L. and Blau, H.M. 2005. Argonaute 2/RISC resides in sites of mammalian mRNA decay known as cytoplasmic bodies. Nat. Cell Biol. 7: 633-636.

Stark, A., Brennecke, J., Bushati, N., Russell, R.B., and Cohen, S.M. 2005. Animal microRNAs confer robustness to gene expression and have a significant impact on $3^{\prime}$ UTR evolution. Cell 123: 1133-1146.

Temme, C., Zaessinger, S., Meyer, S., Simonelig, M., and Wahle, E. 2004. A complex containing the CCR4 and CAF1 proteins is involved in mRNA deadenylation in Drosophila. EMBO $\mathrm{J}$. 23: 2862-2871.

Valencia-Sanchez, M.A., Liu, J., Hannon, G.J., and Parker, R. 2006. Control of translation and mRNA degradation by miRNAs and siRNAs. Genes \& Dev. 20: 515-524.

Wu, L., Fan, J., and Belasco, J.G. 2006. MicroRNAs direct rapid deadenylation of mRNA. Proc. Natl. Acad. Sci. 103: 40344039 . 


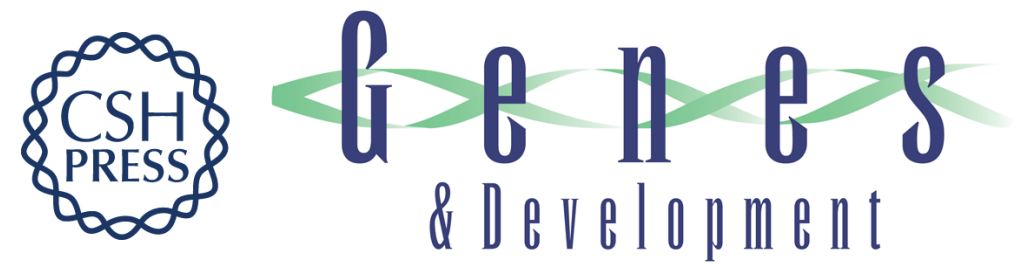

\section{mRNA degradation by miRNAs and GW182 requires both CCR4:NOT deadenylase and DCP1:DCP2 decapping complexes}

Isabelle Behm-Ansmant, Jan Rehwinkel, Tobias Doerks, et al.

Genes Dev. 2006, 20:

Access the most recent version at doi:10.1101/gad.1424106

\section{Supplemental http://genesdev.cshlp.org/content/suppl/2006/06/30/gad.1424106.DC1 Material}

References This article cites 32 articles, 16 of which can be accessed free at: http://genesdev.cshlp.org/content/20/14/1885.full.html\#ref-list-1

\section{License}

Email Alerting

Service

Receive free email alerts when new articles cite this article - sign up in the box at the top right corner of the article or click here.

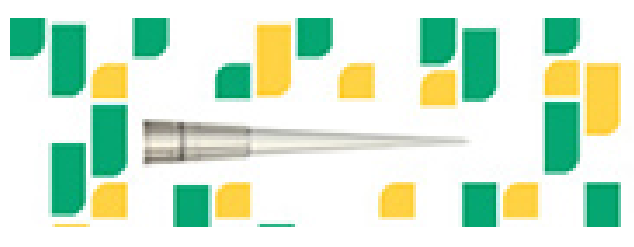

Focused on your science. 